\title{
1. Scholarship, knowledge, concepts
}

A book about scholars needs to start with a basic sense of what scholarship is. This is not an easy task as it would set us on a difficult path in seeking precision about what knowledge is, about whether there are different types of knowing and about whether knowledge is derived for its own sake or is entwined with other purposes. I will start with the simplest possible sense, trying to stay clear of the slippery edges, before mustering the courage to peek over them.

\section{SCHOLARSHIP AND ITS THREE ROLES}

Scholarship implies devotion to (academic) study of a particular subject, whereby one develops over time an expertise or deep knowledge of that subject. Such knowledge is typically conceptual in nature - as opposed to the practical, embodied knowledge one develops as a practitioner - and it enables the expert scholar to highlight key essences and relationships of the subject area, expressed in abstract categories of thought. The major activity of a scholar is thus to think. Thinking involves finding a level of abstraction that enables us to grasp the essence of the entities, situations, processes and events that the subject area encompasses. We typically use the terms 'scholar' and 'academic' interchangeably, and see the place of an academic in a university or other institution of higher education.

Within a university an academic operates at three distinct interfaces, captured by the familiar workload models that 
exist across universities, albeit in different forms: research, teaching and service (or engagement). In the broadest terms, research is about the generation of new knowledge. Teaching is about the codification and transfer of such knowledge to others; it is fundamental to the transmission and learning of social practices. Service is about a contribution to the operation of the university, region or society.

Each of these activities has distinct practices that define what constitutes good performance. In research, they relate to standards of impact, originality and rigour. In teaching, it is about specifying learning outcomes and applying pedagogical principles. In service and engagement more broadly, it is about attuning to the problems, language and practices of particular communities. Through their distinct practices, the three activities pull the academic in different directions. Yet, they also keep one another in check, thereby creating an adaptive system for engaging with the world. The essence of scholarship thus lies in the ability to operate at all three interfaces, not losing sight of the broader system. The reality of academic life, however, is that when the relative status of the three activities becomes disbalanced - with research typically gaining the upper hand - people tend to specialize in or prioritize one of these activities. This creates a vicious cycle whereby emphasis on research diminishes concerns for pedagogy and isolates the academic from the communities of practitioners.

\section{NUANCES IN KNOWLEDGE AND SUBJECT AREA}

Let us consider for a moment two persons totally devoted to the subject of football (soccer): one is immersed in playing it and the other in observing it and commentating on it. They both develop deep knowledge of football: for one, the knowledge is practical in nature, such as a tacit ability to control, pass and shoot the ball in various ways; for the other the 
knowledge is conceptual or theoretical, creating categories that distinguish different ways of controlling, passing and shooting and describe football as a type of sports game. In this sense, both persons are experts in the subject.

Conceptual knowledge is fundamental to being human - it is indispensable in making sense of and coping with the world around us. It implies an observational stance, that is, it poses an object of observation or reference that is external to a focal observer. To the extent that such knowledge is to make sense to humans, the focal observer is always a human person. Thus, conceptual knowledge implies a human-centric stance or perspective that is directed at what we naturally perceive as an external world, that is, a world observed from a distance.

In contrast, in some cases, the question of practical knowledge does not arise at all. Because practical knowledge - in a way that would make sense to us - relates to what a human person does, it presupposes that a human is engaged in the doing of what is being studied. In this sense, it is helpful to differentiate subjects into those that involve human tasks or functions, that is, when humans are performers of or in the phenomenon to be studied, and those that involve natural phenomena (of which the human is always an external observer and thus not part of the phenomenon, at least at the level of human consciousness ${ }^{1}$ ). Human subjects thus span the arts, humanities and social sciences, in which one can be both an external observer and a purposeful internal participant. It is thus only in these subjects that practical knowledge arises, and thereby its distinction from theoretical knowledge.

When both theoretical and practical knowledge are possible, a person can take a dual stance of observer and participant. This creates a self-referential loop whereby the person refers (as observer) to what the person does (as participant),

1 Humans are clearly part of the natural world in the sense that a human is a biological system. But at the level at which our consciousness operates, this system is not directly observable. 
and what the person does reflects what the person sees. This loop helps to keep theoretical and practical knowledge in one coherent whole, whereby each conceptual category that arises can be easily exemplified with practical actions, and each practical action can be described in categorical terms. But when this self-referential loop is severed, as when observer and participant are segregated, theoretical and practical knowledge can grow disconnected.

Practical knowledge can be distinguished further, using Aristotle's (1999) distinction of the intellectual virtues of episteme, techné and phronesis. For Aristotle, the intellect has a scientific part that contemplates things with invariable causes and a calculative or practical part that deals with the contingencies of everyday life. Such contingencies comprise things made (making) and things done (acting) and their truth is relative to purpose and values. Therefore, episteme refers to universal truths or categories as theoretical, value-free knowledge. Techné arises in the making of things and thus refers to art and craft. Phronesis arises in judgement and action and thus refers to practical wisdom.

A simple insight from this distinction is that if we take a scholarly interest in human life with its everyday contingencies, we need not only to focus on making sense of the world overall but also to be sensitive to making and acting as distinct domains of knowledge and learning. Thus, to go back to the earlier example, when a third-person comes along, who wants to learn to play football, the knowledge of both experts becomes indispensable. We need the bodily skill and intuitive understanding of the game that is offered by the practical expert (which in this example combines making and acting), but we also need the conceptual schema of the theoretical expert in order to develop a deeper, more expressive understanding of the game and appreciate its broader purpose and place in society. In reality, when theoretical and practical knowledge are disconnected, they become disjointed loci of 
learning, with some learners looking exclusively for practical knowledge and others exclusively for theoretical.

\section{SOME FUNDAMENTALS OF CONCEPTUAL KNOWLEDGE}

We can now delve into thinking as an activity that involves finding a level of abstraction that enables us to grasp an essence in or articulate the meaning of what is observed. Thinking creates a relation between a person (the scholar) and an external object or activity (that is, as comprising the subject of study). What results from this process is knowledge that constitutes a mental representation (that is, concept) of the external object that in turn needs to be expressed in some symbolic form, such as verbal or mathematical language. Such expression enables knowledge to be communicated, shared, corroborated, refined or challenged. Thus, the knowledge arising from scholarship is an explicit product of intellectual effort that can be consumed by others. It communicates concepts via words and other symbols.

We identify scholarly knowledge by its symbolic expression, yet we also understand that the symbols used stand for something else: for concepts they are meant to express and, ultimately, for entities, processes or events in the world around us that are represented by such concepts. Our everyday language is the simplest example of such symbolic representation, whereby with the words we use we can invoke in our minds entities, process or events, real or imaginary. In other words, a language - of any form - is indispensable for expressing our thoughts. The essential nature of language is that its expressions can be understood by other people, that is, the particular community who use it to communicate.

When it comes to conceptual knowledge, we thus encounter an interplay between a focal individual, a community of other individuals - each of whom can also be a focal individual - and an external world, that is, what is outside of 
the focal individual. This interplay is elegantly expressed in Donald Davidson's (2001) notion of the knowledge tripod. It highlights three distinct varieties of knowledge, each based on a distinct mode of access to the same all-encompassing reality. These are subjective (first-person), intersubjective (second-person) and objective (third-person) knowledge. They are not reducible to one another and, in this sense, the notion of tripod suggests that 'if any leg were lost, no part would stand' (p.220). From our own subjective, first-person point of view, to express our thoughts we need a language. To have a language, we need to know other minds (a second-person), to make sure that whatever symbolic expression we use will be understood as such by others. To know other minds, we need a shared external world against which to triangulate meaning, to ensure that the symbols we use stand for something that is commonly perceived and similarly understood.

The interplay among the legs of the tripod becomes evident when we consider something as simple as a rock (in our everyday language). It is a seemingly simple object, but it can be described in many different languages as well as through many different categories or conceptual schemas. Its sense of an everyday object can be conveyed in different foreign language terms (piedra, Stein, камък, камень, and so on), all with the same meaning to be conveyed within the community of speakers of those languages. But it can be described as a molecular system, chemical composition, geological structure, object of worship, tool (such as a paper weight or nutcracker), art object, pet and so on. Similarly, a person can be described as a multicell organism; a nervous, circulatory or respiratory system; an entrepreneur; a parent; a manager; and so on. Each of these descriptions ushers us into a specific community of others, united by some common interest or activity and sharing symbolic expressions that reflect that interest or activity. Therefore, to state what something 'is' is to aim to express a particular point of view, find a community 
of others who share the same point of view and use the language the community shares.

In this sense, scholarship - and particularly science exerts particular care and precision in defining the reality it is to study and calibrating the language used to describe it. A simple everyday object or activity can thus be the subject of many different areas of scholarship. The depth of knowledge that scholarship produces is enabled by the reality frame it uses to define the 'object' of scholarship. Such frame defines the essential and nonessential properties of what is to be studied. A frame thus implies a clear categorical boundary and, as such, it makes clarity and precision possible. In other words, scholarship implies a commitment to a particular way of seeing the world, using a particular set of categories of interest. This is reflected in the tree of knowledge system that distinguishes four dimensions of complexity, namely matter, life, mind, culture (Henriques 2008). Associated with these are four broad classes of science - physical, biological, psychological and social - each of which contains finer divisions into specific domains of scholarship.

The language of each domain is its theory. It defines the basic concepts that comprise the domain and enables these concepts to be communicated among the members of the community. In this sense, domain and theory go hand in hand. What makes a domain distinct is its ontology, that is, a foundational sense of what it is, identifying the basic building blocks or essential things of which it is made. Thus, whether a person is seen as made of cells or is seen as made of interpersonal relationships represent different domains of interest and, accordingly, different ontologies. The basic building blocks are categories of objects (used in a generic sense to include entities, events or processes) that define essential properties as relevant for the domain. They can be weaved together into a distinct conceptual model that can be used as a tool for understanding and coping with the world. 
In this way, theory as the distinct language of the domain gives external objects a domain-specific meaning, even if it uses expressions from common language. In the light of this, Quine (1960[2013]) portrays science as an extension of common language: not separate from it, but a regimented version of it. It brings clarity through the reference objects it postulates through its conceptual scheme. Thus, the question of what there is (ontology) is very much part of the scientific endeavour: 'What reality is like is the business of scientists, in the broadest sense, painstakingly to surmise; and what there is, what is real, is part of that question.' (Quine, 1960[2013], $\S 581)$.

\section{MAP AND TERRITORY}

To be a scholar, then, is to be a scholar of something, to make a commitment to a particular ontology, a particular set of conceptual categories that become basic building blocks from which to build understanding of some aspect of the world. This is just like working with different Lego sets - each consisting of different basic blocks, some square, others round - from which to build models of the world. There is an initial choice of what set to use. It creates a natural community of likeminded users, sharing common set pieces. Thus, it makes little sense for someone using square pieces to admonish another person committed to using round pieces: they belong to different theoretical communities. They know different things.

But what does it mean to know something? The Nobel Prize winning physicist Richard Feynman famously drew the distinction between knowing the name of something and knowing something. In a 1973 TV documentary, he makes two profound points: (1) names do not constitute knowledge; and (2) names are useful when communicating with others. This suggests that, in our quest for understanding, there are two distinct, yet interrelated and complementary steps. The 
first involves naming something and thereby making it an explicit focus of scholarly study. For example, social entrepreneurship directs attention to business activities that are motivated by social impact. The second involves describing its nature and operation in terms of the basic language of the scholarly domain. The two activities go hand in hand, since a new name can be justified by distinct configuration of the representational elements, and vice versa.

Figure 1.1 summarizes the points made so far. It distinguishes a realm of practice made of what we can generically refer to as objects of attention - things we sense, make, do in the world - and a realm of theory made of concepts, that is, words and other symbols that stand for objects in the world. Scholarship represents an engagement with the world via concepts: the practical interests of the scholar define the aspect of the world to be represented by concepts via a process of denotation. In turn, the concepts relate to the world via a process of reference, whereby objects in the world are described, expressed or perceived by those concepts, within the theoretical framework that defines them. The combination of objects and concepts create a realm of meaning as an interplay of denotation and reference. Meaning changes when the same concepts are used to refer to new objects or when the same objects are denoted by new concepts.

Portrayed in these terms, it is clear that knowledge is a sort of map of something, of some territory of the broader world, which to an uninitiated observer (such as an alien or a newborn) may appear as an incomprehensible mess. Thus, to know something is to be able to construct a (conceptual) map of it. But deeper questions arise when we consider Korzybsky's (1933) famous claim 'the map is not the territory'. In its elaboration, Korzybsky outlines two crucial negative premises: (1) words are not the things we are speaking about; (2) there is no such thing as an object in absolute isolation. The first relates to the earlier discussion of the different ways to describe something; no description is exhaustive. There are different 


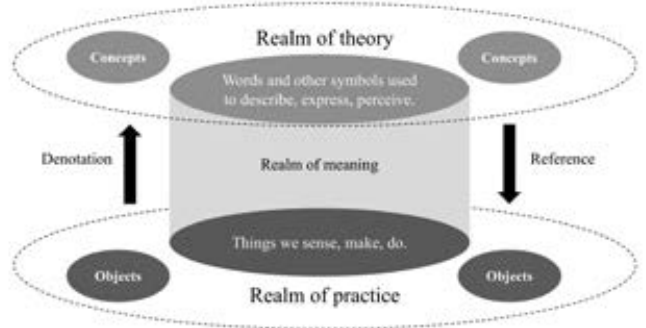

Figure 1.1 Denotation and reference

maps possible of the same territory. The second relates to the earlier point that each description requires a frame that isolates the object to be described in terms of essential and nonessential features.

If a map is not the territory it represents, then the question arises of what gets on the map. Bateson (1972) articulates that what gets on the map is difference, and difference is an abstract matter. It is not an inherent property of things but is attributed to them by an observer who puts things side by side and draws a comparison. For example, when looking at two people, we can draw differences in terms of height, weight, age, biological sex, gender, language, nationality, profession, marital status, knowledge of the world's capital cities, ability to draw flowers, and so on. There are an infinite number of differences we can highlight in this case. Each difference is related to the kind of map we would like to build, or in Kantian terms, the facts we would like to select and make relevant. In this sense, Bateson concludes that an idea in its most elementary sense is synonymous with difference. Information is a difference that makes a difference. 
What we put on the map are ideas. And ideas arise from a fundamental function of our mental processes, namely to distinguish. In light of the latter, Korzybsky highlights two main tendencies in science. The first is to conduct experiments in order to detect differences. Associated with this is the design and improvement of instruments that can detect such differences. The second is critical verbal rigour, associated with the invention of better forms of representation (that is, theories). As already intuited, both are critically important: isolated facts do not make a science; they need to be brought together into a structure in the form of theory. Actions (making of facts) and concepts (structuring of facts) are thus complementary and indispensable to one another.

\section{CONCEPTS AS MEDIATORS OF BEHAVIOUR}

Concepts are essential for facilitating learning and thus control of behaviour. Understanding the instrumental role of concepts rests on a seminal idea by Vygotsky, who suggests that the use of signs (symbols) acts as a mediator of behavioural acts and thus enables behavioural control.

Because this auxiliary stimulus possesses the specific function of reverse action, it transfers the psychological operation to higher and qualitatively new forms and permits humans, by the aid of extrinsic stimuli, to control their behaviour from the outside. The use of signs leads humans to a specific structure of behaviour that breaks away from biological development and creates new forms of a culturally-based psychological process. (Vygotsky 1978, p.40)

Signs can thus act as artificial stimuli in a given situation and can serve as immediate causes of behaviour.

This happens through the role of signs as conduits for meaning. Vygotsky highlights that as abstract thought develops, meaning begins to dominate action in the sense that 
action plays an instrumental role in an imaginary world of aspirations, goals and values. Imagination is a specifically human form of conscious activity that enables humans to rise above situational constraints: in an imaginary situation, it is not the immediate perception of objects that guides action, but the meaning of the situation. In this way, action becomes a pivot through which a person can move in a field of meaning. Perception and meaning begin to diverge once children begin to separate objects from the concepts used to describe them. In this way, play emerges as the creation of imaginary situations in which thought becomes separated from objects. Thought acquires causal power because it infuses meaning.

When the use of signs is viewed in the context of cultural evolution, concepts stand out through the ways they help our thinking, improving our discrimination and memory as well as becoming a storehouse of cultural evolution and social learning (Henrich 2016). Concepts or categories enable us to make sense of the world and deal with new situations by treating them as familiar. The creation and evocation of concepts is driven by the making of analogies and this represents the core of our thinking: 'for without concepts there can be no thought, and without analogies there can be no concepts' (Hofstadter and Sander 2013, p.3). Categories are evolving mental structures that contain information in an organized form, which can be accessed via acts of categorization, whereby a new situation can be seen and understood in a particular way.

This idea is linked and reinforced by Sellars's (1963) refutation of the 'myth of the given', that is, the idea of immediate empirical knowledge. For Sellars, classificatory consciousness - the claim that something is thus and so - involves learning, concept formation and the use of symbols: "for now we recognize that instead of coming to have concept of something because we have noticed that sort of thing, to have the ability to notice a sort of thing is already to have the concept of that sort of thing, and cannot account for it' (p.176). 
There is thus a creative act or mental leap when existing categories are applied to new situations or analogies are made to derive new concepts. Hofstadter and Sander provide the example of Galileo's observation of Jupiter after constructing his first telescope, at a time when there was no sharp distinction between stars and planets. An initial surprise was that, while Jupiter appeared as a dot when seen with a naked eye, it became a small circle when seen through the telescope. Using the analogy of someone approaching you with a lantern directed towards you (where the lantern grows from a dot to a growing circle), Galileo concluded that Jupiter was a physical object. He then observed tiny black dots against the white background of the circle, moving across in a straight line. When reaching the edge of the white circle, they became white against the black background of space and continued their straight-line motion, before slowing down to a stop, reversing their motion, and disappearing when reaching the edge of the white circle, only to reappear later on at its other side. Galileo knew that the Earth was round and that the Moon rotated around it periodically, but Earth and Moon were the proper names of single objects. Galileo's mental leap was to pluralize these and thus 'see' a second 'Earth' in the sky with several 'Moons'. This gave rise to the concepts of planet and moon. From today's point of view - when these concepts are taken for granted - it is difficult to imagine that there was a time in which what the dots in the sky were had not been given.

Bringing all the strands of thought together, we started with the general idea of the scholar as a thinker and we can now distinguish four distinct strands of the activity of thinking, as illustrated in Figure 1.2. They form a cycle that reflects the interplay of theory and practice.

(1) Generation and detection of differences in the territory (practice). This includes both empirical research and actual practice. 
(2) Generation of new concepts to name and communicate these differences.

(3) Organization and relation of concepts to create a map of the territory (theoretical work).

(4) Application of concepts to the territory - to frame and give meaning to new areas, situations or aspects of it. This includes both the natural situations of practice and the generation of an artificial situation, such as when conducting research via a survey or experiment.

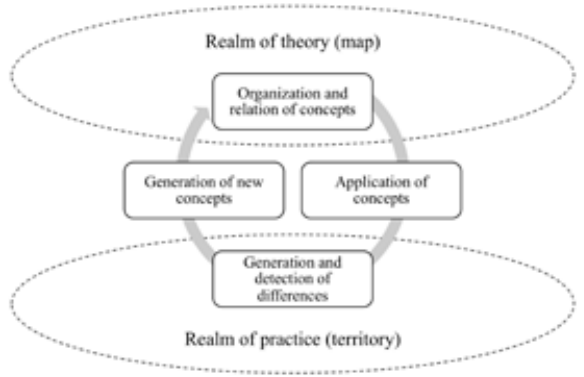

Figure 1.2 Cycle of thinking

This cycle of thinking can be related more broadly to Piaget's (1950) theory of cognitive development, based on an interplay between assimilation and accommodation. Assimilation entails perceiving new objects through existing mental schemas, while accommodation entails adapting the schema to incorporate ill-fitting objects or situations. This idea also forms the basis of experiential learning theory (Kolb 1984). It specifies an overall learning cycle, in which new schemas 
arise and operate through reflective observation and abstract conceptualization, and are then applied and enacted through active experimentation and concrete experience.

Having explored so far the nature of knowledge as a sort of map of some territory, in the next chapter I will explore the specific territory or domain of entrepreneurship. 